\title{
Associative Conditioning Tunes Transient Dynamics of Early Olfactory Processing
}

\author{
Patricia C. Fernandez, ${ }^{\star}$ Fernando F. Locatelli, ${ }^{*}$ Nicole Person-Rennell, Gregory Deleo, and Brian H. Smith \\ Arizona State University, School of Life Sciences, Tempe, Arizona 85287-4501
}

Odors evoke complex spatiotemporal responses in the insect antennal lobe $(\mathrm{AL})$ and mammalian olfactory bulb. However, the behavioral relevance of spatiotemporal coding remains unclear. In the present work we combined behavioral analyses with calcium imaging of odor induced activity in the honeybee AL to evaluate the relevance of this temporal dimension in the olfactory code. We used a new way for evaluation of odor similarity of binary mixtures in behavioral studies, which involved testing whether a match of odor-sampling time is necessary between training and testing conditions for odor recognition during associative learning. Using graded changes in the similarity of the mixture ratios, we found high correlations between the behavioral generalization across those mixtures and a gradient of activation in AL output. Furthermore, short odor stimuli of $500 \mathrm{~ms}$ or less affected how well odors were matched with a memory template, and this time corresponded to a shift from a sampling-time-dependent to a sampling-time-independent memory. Accordingly, $375 \mathrm{~ms}$ corresponded to the time required for spatiotemporal AL activity patterns to reach maximal separation according to imaging studies. Finally, we compared spatiotemporal representations of binary mixtures in trained and untrained animals. AL activity was modified by conditioning to improve separation of odor representations. These data suggest that one role of reinforcement is to "tune" the AL such that relevant odors become more discriminable.

\section{Introduction}

Odor stimulation evokes spatial patterns of activity in the insect antennal lobe $(\mathrm{AL})$ and mammalian olfactory bulb $(\mathrm{OB})$ that covary with odorant molecular structure (Sachse et al., 1999; Wang et al., 2003; Lei et al., 2004; Johnson and Leon, 2007), mixture composition (Giraudet et al., 2002; Duchamp-Viret et al., 2003; Heinbockel et al., 2004; Tabor et al., 2004; Silbering and Galizia, 2007) and concentration (Johnson and Leon, 2000; Meister and Bonhoeffer, 2001; Sachse and Galizia, 2003; Stopfer et al., 2003; Hallem and Carlson, 2006; Silbering et al., 2008). However, activity also consists of fast and slow temporal patterns that correlate to odorant features (Kashiwadani et al., 1999; Laurent et al., 2001; Laurent, 2002; Daly et al., 2004a; Friedrich et al., 2004). Several studies have shown that temporal processing can make initially similar activity patterns more distinct over longer sam-

\footnotetext{
Received April 13, 2009; revised June 18, 2009; accepted July 8, 2009

This work was supported by grants from National Institutes of Health (NIH)-National Center for Research Resources (NCRR RR014166) and NIH-National Institute on Deafness and Other Communication Disorders (R01 DC007997) to B.H.S. We thank Giovanni Galizia for the training in the calcium imaging technique and providing customized software. Tanja Bloss measured EAG signals. We also thank Julie Mustard and Matthiew Dacher for comments at an early stage of this manuscript and Mathias Ditzen for providing software for the glomerular identification.

*P.C.F. and F.F.L. contributed equally to this work.

Correspondence should be addressed to Brian H. Smith at the above address. E-mail: brianhsmith@asu.edu.

P. C. Fernandez' present address: Instituto Nacional de Tecnología Agropecuaria, Estación Experimental Delta de Paraná y Facultad de Agronomía de la Universidad de Buenos Aires, Cátedra de Química de Biomoleculas, Departamento de Biologia Aplicada y Alimentos, Avenida San Martin 4453, Ciudad Autónoma de Buenos Aires, Argentina.

F. F. Locatelli's present address: Laboratorio de Neurobiología de la Memoria, Departamento Fisiología, Biología Molecular y Celular, Facultad de Ciencias Exactas y Naturales-Universidad de Buenos Aires, Instituto de Fisiología, Biología Molecular y Neurociencias-Consejo Nacional de Investigaciones Científicas y Técnicas, Intendente Güiraldes 2160 Pabellon II Ciudad Universitaria, C1428EHA Ciudad Autónoma de Buenos Aires, Argentina.

DOI:10.1523/JNEUROSCI.1874-09.2009

Copyright $\odot 2009$ Society for Neuroscience $\quad 0270-6474 / 09 / 2910191-12 \$ 15.00 / 0$
}

pling times (Stopfer et al., 1997; Fdez Galán et al., 2004; Friedrich and Laurent, 2004; Mazor and Laurent, 2005). This processing sets up a series of activity patterns, or "transients," that could improve odor discriminability (Rabinovich et al., 2008). Thus, in principle, more specific information should be available as sampling times increase.

Previous studies aimed at evaluating tradeoffs between sampling time and decision accuracy have shown that longer sampling times can improve performance (Abraham et al., 2004; Rinberg et al., 2006; Wright et al., 2009). However, animals are also capable of making reasonably accurate decisions even with short sampling times (Ditzen et al., 2003; Uchida and Mainen, 2003). Therefore, the issues of specifically when and how time is relevant for odor identification require further investigation.

Furthermore, there is now growing evidence that $\mathrm{AL}$ and $\mathrm{OB}$ neural activity may be altered by plasticity (Davis, 2004). Repeated nonassociative exposure to an odor increases the coherent spiking of projection neurons (PNs) in the locust AL (Stopfer and Laurent, 1999) and changes the receptive fields of mitral cells in the OB (Vanderwolf and Zibrowski, 2001; Fletcher and Wilson, 2003). Several studies in insects have reported that neural activity in the AL is altered by classical conditioning (Faber et al., 1999; Müller, 2000; Sandoz et al., 2003; Daly et al., 2004b; Yu et al., 2004; Thum et al., 2007), and conditioning alters oscillations in specific frequency bands in the mammalian OB (Ravel et al., 2003; Martin et al., 2004; Beshel et al., 2007).

Our study investigated how associative plasticity affects spatiotemporal activity patterns and how both impact behavioral decisions. We combined a robust olfactory conditioning paradigm in the honeybee for evaluating odor perception (Smith et al., 2006; Giurfa, 2007) and in vivo imaging of odor driven activity 
in the AL (Sachse and Galizia 2002). We used binary mixtures that differ in ratios of components at low concentration, which makes discrimination difficult. We found that conditioning increased the separation between the spatiotemporal patterns evoked by the binary mixtures in the AL. Furthermore, the time needed for maximal separation of odor representations corresponded to the time at which honeybees transition from sampling time-dependent to sampling time-independent odor discrimination.

\section{Materials and Methods}

Animals. Forager honeybees (Apis mellifera carnica) were collected in the morning at the entrance of the hive, shortly cooled, and restrained in individual harnesses. After recovering from cooling, bees were fed $2 \mu \mathrm{l}$ of a $1.0 \mathrm{M}$ sucrose solution and allowed to remain undisturbed for $2 \mathrm{~h}$ at room temperature until conditioning took place.

Odor stimulation. Odors used for stimulation were the aliphatic alcohol 1-hexanol and the ketone 2-octanone (both TCI America, 98\%), alone or combined in binary mixtures in the ratios 9:1, 7:3, 5:5, 3:7, and 1:9 (1-hexanol: 2-octanone molar ratio). Odors were diluted from purity in mineral oil (Sigma-Aldrich) and mixed to obtain in all cases a final concentration of $0.02 \mathrm{M}$. The output of the odor delivery device, either in behavioral or imaging experiments, was positioned $2 \mathrm{~cm}$ away from the bee's head and targeted toward the antennae. During the experiment a continuous charcoal filtered air stream $(25 \mathrm{ml} / \mathrm{s})$ ventilated the antennae. Five centimeters behind the bee an exhaust continuously removed the air from the arena. The odor cartridges consisted of $1 \mathrm{ml}$ glass syringe containing a filter paper strip $(0.5 \times 4 \mathrm{~cm})$ loaded with $10 \mu$ l odorant solution. Four $\mathrm{cm}$ upstream from the cartridge, a three-way valve (LFAA1200118H; The LEE Company) controlled the onset of the airflow through the odor cartridge.

In the behavioral experiments, opening of the valve was triggered by a programmable controller (Automation-Direct) and the durations used were: $200,500,1000,2000$, or $4000 \mathrm{~ms}$. In the imaging experiments, the valve was synchronized with the optical recordings directly from the imaging acquisition software TILLVisION (TILL Photonics) and valve opening lasted $1000 \mathrm{~ms}$ except in the experiment plotted in figure S5C. When the valve was open, the odor-laden air from the cartridges was pushed into the continuous air stream in a mixing chamber $2 \mathrm{~cm}$ before the output of the odor delivery device. The odor device had eight independent and identical channels composed of a valve and an odor cartridge. Calculation based on the geometry of the odor delivery device and the airflow yielded an estimated delay of $15 \mathrm{~ms}$ between opening of the valve and the odor reaching the antennae. The valve response time provided by the manufacturer was as fast as $0.25 \mathrm{~ms}$. Between experiments, odors were rotated across channels to balance any differences between channels.

Olfactory conditioning. A differential conditioning protocol was used to train bees and examine their ability to perceive differences among odors. Bees were trained as described by Smith (1998). Each bee received differential conditioning to two odorants in the following pseudorandomized 12 or 16 trial sequence: $A B B A B A A B A B A B$ or $A B B A B A A B A B A B B A B A$ respectively, where $A$ is the rewarded odor and $B$ is the unrewarded one. The intertrial interval was $6 \mathrm{~min}$. In the rewarded trials $(\mathrm{CS}+)$, odor was paired with the unconditioned stimulus (US), which consisted of first touching the antennae with a $2 \mathrm{~m}$ sucrose solution and followed by feeding with $0.4 \mu \mathrm{l}$ during extension of the proboscis. In unrewarded trials (CS-), bees were stimulated with odor but sucrose was omitted. Duration of odor stimulation ranged from 200 to $4000 \mathrm{~ms}$ depending on the experiment (see Figs. 1-6). The US was omitted on tests trials and the odorants were presented as above. To avoid extinction of the conditioned response, a retraining trial was offered every 2 test trials. During the retraining trial, the CS+ was paired with the US whereas the CS- was not, in the same way as was done during the training phase.

On every acquisition trial, the response of each subject was recorded as a positive response if the subject extended its proboscis during odor stimulation and before the US presentation. In the experiments in which the odor sampling time was $4000 \mathrm{~ms}$, the sucrose US was presented $3 \mathrm{~s}$ after the start of odor stimulation, which allowed for $1 \mathrm{~s}$ overlap between odor and the US. In the experiment with variable sampling time (see Fig. 6) the US was presented in all cases $1 \mathrm{~s}$ after odor onset. Thus, there was no CS-US overlap for 200 and $500 \mathrm{~ms}$, there was $1 \mathrm{~s}$ overlap for $2000 \mathrm{~ms}$ and, in the case of $1000 \mathrm{~ms}$, the start of the US coincided with the odor shut off. These data were plotted as the percentage of subjects that responded to the odor on each trial. Proboscis extension was recorded as a binary variable - extension response or not—-during the training trials. Each test trial was also recorded using a digital camcorder for offline quantification of the response duration. Duration of the proboscis extension was used as a response measure because it has been shown that it is more sensitive for revealing differences in response among treatment groups than is a percentage response (Smith and Menzel 1989, Smith 1997). Duration is defined as the elapsed time that the proboscis is extended beyond the line connecting the tips of the opened mandibles (as the subject is viewed along the longitudinal axis).

Statistical analysis. Duration of proboscis extension was analyzed by a two way repeated measures ANOVA using a generalized linear model (GLM) (SPSS) and GLM a priori contrasts. For the timing experiment, we used specific pairwise comparisons (CS+ vs CS-) by Wilcoxonmatched pairs test.

Calcium imaging. Bees were restrained into Plexiglas stages suited for proboscis extension response (PER) conditioning and optical recordings. The head was fixed to the stage with soft dental wax (Kerr, Sybron Dental Specialties) in a way they could freely move antennae and proboscis. Nine to ten hours after behavioral conditioning, PNs were stained by backfilling with the calcium sensor dye fura-dextran (potassium salt, $10.000 \mathrm{MW}$, Invitrogen). A window was cut in the head capsule dorsal to the joints of the antennae and rostral to the medial ocellus. The glands were carefully moved until the $\alpha$-lobes (Rybak and Menzel, 1993) in the brain were visible, which is easily recognizable and serves as spatial reference for the staining (Sachse and Galizia, 2002). The tip of a glass electrode coated with fura-dextran prepared in $3 \%$ bovine serum albumin solution (Sigma-Aldrich) was inserted into both sides of the deutocerebrum dorsolateral to the $\alpha$-lobes, aiming for the lateral antennocerebral tracts (1-ACT) that contains the axons of a subset of uniglomerular PNs (Abel et al., 2001). The dye bolus dissolved into the tissue in $3-5 \mathrm{~s}$ and the window was immediately closed using the same piece of cuticule that was previously removed. Eicosane was used to glue and seal the cuticule. The dye was left to travel along the tracts for $8-12 \mathrm{~h}$.

Before imaging, the antennae were fixed pointing toward the front using eicosane (Sigma-Aldrich) and body movements were prevented by gently compressing the abdomen and thorax with a piece of foam. The brain was rinsed with Ringer solution ( $130 \mathrm{~mm} \mathrm{NaCl}, 6 \mathrm{~mm} \mathrm{KCl,} 4 \mathrm{~mm}$ $\mathrm{MgCl} 2,5 \mathrm{~mm} \mathrm{CaCl}$ 2, $160 \mathrm{~mm}$ sucrose, 25 mm glucose, $10 \mathrm{~mm}$ HEPES, $\mathrm{pH}$ 6.7, $500 \mathrm{mOsmol}$; all chemicals from Sigma-Aldrich), and glands and trachea covering the antennal lobes were removed. A second hole was cut ventrally to the antennae and the compact structure of muscles, esophagus, and supporting chitin was lifted and put under slight tension to prevent movements of the brain (Mauelshagen 1993). Thus, stabilization was accomplished without damage to the brain. After surgery and before measurements bees were allowed to recover for $20 \mathrm{~min}$. The antennal lobes were examined for appropriate staining and only the one that presented the more homogenous staining was selected for the measurements.

Calcium imaging was done using a CCD camera (SensiCamQE, T.I.L.L. Photonics) mounted on an upright fluorescence microscope (Olympus BX-50WI, Japan) equipped with a $20 \times$ dip objective, numerical aperture $=0.95$ (Olympus), 505 DRLPXR dichroic mirror, and 515 $\mathrm{nm}$ LP filter (TILL Photonics). Monochromatic excitation light provided by a PolichromeV (TILL Photonics) alternated between 340 and $380 \mathrm{~nm}$. Fluorescence was detected at a sampling rate of $8 \mathrm{~Hz}$. Spatial resolution was $172 \times 130$ pixels, after a binning of $8 \times 8$ on a chip of $1376 \times 1040$ pixels, resulting in a spatial sampling of $2.6 \mu \mathrm{m}$ per pixel side. Exposure times were 8 and $2 \mathrm{~ms}$ for 340 and $380 \mathrm{~nm}$ respectively.

The entire image analyses were done using custom software written in Interactive Data Language (IDL; Research Systems) created by Giovanni 
Galizia and Mathias Ditzen (University of Konstanz, Konstanz, Germany). Each data set or measurement consisted in a double sequence of 80 fluorescence images, obtained at 340 and $380 \mathrm{~nm}$ excitation light $\left(F i_{340}, F i_{380}\right.$, where $i$ is the number of images from 1 to 80$)$. Calcium signals were subsequently calculated using a ratiometric method: For each pair of images $F i$ we calculated the ratio $R i=(F i 340 \mathrm{~nm} / F i 380$ $\mathrm{nm}) \times 100$ and subtracted the background $R_{b}$, obtained by averaging the $R i$ values $1 \mathrm{~s}$ immediately before the odor onset $\left[R_{b}=1 / 8\left(R_{16}+\ldots+\right.\right.$ $R_{23}$ )]. Resulting values represent percentage of change from the reference window $\left(R_{16}-\mathrm{R}_{23}\right)$ and are proportional to the changes in the intracellular calcium concentration. The analysis was based on the time course of calcium signals in each identified glomerulus. For this aim, glomeruli were identified on the basis of their morphology and relative position using the digital atlas of the honeybee $\mathrm{AL}$ as a reference (Galizia et al., 1999). The visualization of glomeruli is possible observing the raw fluorescence images obtained at $380 \mathrm{~nm}$ excitation light. An additional tool written and provided by Mathias Ditzen was used to confirm glomerular shape and position. This tool calculates images representing the degree of correlation between neighboring pixels. Since glomeruli respond as functional units, pixels stemming from the same glomerulus are highly correlated over time. In contrast, pixels from different glomeruli are uncorrelated. This provides images in which glomeruli are clearly visible and clearly separated by contrasting boundaries.

Seventeen glomeruli were identified across all animals and were used in the present analysis. All glomeruli were located in the dorsorostral side of the AL and correspond to a subset of glomeruli innervated by the antennal nerve tract 1 (Kirschner et al., 2006). We calculated activity in glomeruli 17, 23, 24, 25, 28, 29, 33, 35, 36, 37, 38, 42, 43, 47, 48, 49, and 52 according to the nomenclature previously established (Flanagan and Mercer, 1989; Galizia et al., 1999). Glomerular activation was calculated by averaging activity in a square area of $9 \times 9$ pixels that correspond to $23.4 \times 23.4 \mu \mathrm{m}$ and fits well within the boundaries of the glomeruli. Glomerular activity in the present study refers to activity of the uniglomerular PNs in each glomerulus, because only these neurons were stained, and therefore only this particular population of cells was measured in each glomerulus.

For visualization of the data, we reduced the 17 dimensions using a principal component analysis (PCA) that identifies orthogonal axes (factors) that explains maximum variance in the data, and thus projects the data into a lower-dimensionality space. The first two factors that explain most of the observed variance were further subjected to Varimax rotation (SPSS). Such rotational strategy maximizes the variance accounted for by the calculated factors, while minimizing the variance around them.

Statistical analysis was based on euclidean distances (EDs) among pairs of odors calculated in a 17-dimensional space in which each axis represents the activation of one glomerulus $\left(\mathrm{ED}_{\text {hex-oct }}=\left[\left(R_{17 \text { hex }}-\right.\right.\right.$ $\left.\left.R_{17 \text { oct }}\right)^{2}+\ldots+\left(R_{52 \text { hex }}-R_{52 \text { oct }}\right)^{2}\right]^{1 / 2}$, where for example $R_{17 \text { hex }}$ means the response of glomerulus 17 during stimulation with 1-hexanol). This spatial relationship provided us a measure of difference or similarity between the neural representations of two odors. ED was calculated for each possible pair of odors and for the 80 time intervals that lasted each measurement. This provided a matrix of $21 \times 80$ EDs per animal. Since ED between two neuronal representations may be affected by the general strength of the calcium signals, all EDs obtained for each animal were normalized by dividing by the ED obtained for the 10:0-0:10 (hexanoloctanone) pair $500 \mathrm{~ms}$ after odor onset. The normalization based on EDs used the global activity in the AL and avoids normalizing to activity of specific glomeruli that eventually may be not reliable. The latter alternative was, however, considered and is further explained in the supplemental Figure S3, available at www.jneurosci.org as supplemental material, as a complementary analysis. All statistical analysis of EDs was performed using Mann-Whitney $U$ test at each time point for each odor pair.

\section{Results}

\section{Transitions in ratios of binary mixtures define smooth} transitions in odor perception

The goal of this first series of experiments was to establish whether smooth transitions among ratios in binary mixtures would generate a smooth transition in generalization among the odors. The pattern of response- " generalization"-from conditioned odors to novel, unexperienced odors depends on the specific conditioning procedure (Wright et al., 2008). Therefore, high levels of generalization cannot be equated with lack of discriminability, per se. However, for any given conditioning procedure generalization is a function of perceptual similarity, and hence it is a measure of discriminability (Smith and Menzel, 1989).

We used differential conditioning in which animals were conditioned to two ratios; one ratio (the $\mathrm{CS}+$ ) was reinforced with sucrose, whereas the other ( $\mathrm{CS}-$ ) was not. We increased the difficulty of the conditioning task in three ways. First, we used low odor intensities that compromise odor discrimination (Wright and Smith, 2004). Second, instead of using pure odorants that differ in molecular structure, we manipulated the ratios of components in binary mixtures (Ditzen et al., 2003; Uchida and Mainen, 2003; Abraham et al., 2004; Rinberg et al., 2006). Third, we selected mixture components (1-hexanol and 2-octanone) that show partially overlapping patterns of activation in the honeybee AL (Sachse et al., 1999).

Figure $1 A$ (left) shows the mean response probabilities of the PER during conditioning to 1-hexanol (i.e., 10:0) as the CS+ and 2-octanone as the CS- (0:10 mixture) and vice versa (as a convention, 1-hexanol is always shown left of the colon and 2-octanone right). In both experiments, some animals responded spontaneously to the CS+ on the first trial, which is normal for PER experiments (Menzel, 1990). Response to the CS+ increased in each case on subsequent trials, which reflects associative conditioning (Bitterman et al., 1983). The response on the first trial with the CS- (trial 2 of the sequence) was high because of generalization from the previously reinforced trial. On subsequent trials the response becomes more specific to the CS+.

Figure $1 A$ (right) shows the duration (see Materials and Methods) of the response during the test phase. Bees responded longer to the CS+ than to CS - . Therefore these odors (albeit pure odors in this case) are discriminable. There was also a smooth generalization from pure odors to the mixture ratios 1:9 and 9:1. The strongest response was to the mixture closest to the $\mathrm{CS}+$. Repeated measures ANOVA revealed significant interaction between treatments (interaction $p<0.001$ ), which suggests bees were able to discriminate accurately between 1-hexanol and 2-octanone when they each were the CS+.

In a different set of experiments, we differentially conditioned bees to 9:1 and 1:9 mixtures as the CS+ and CS - . Figure $1 B$ (left) shows the mean response probabilities during conditioning. The response to the CS + and $\mathrm{CS}-$ diverged over trials, and the response to the CS+ was consistently higher. In both cases, there was significant divergence in response on the last two trials, which occurred as retraining trials during the testing phase. This pattern indicates that the mixtures are discriminable, but bees have greater difficulty in discriminating these odor ratios that they did in the previous experiment with pure odors.

During the test phase (Fig. $1 B$, right), the duration of the proboscis extension response was higher to the $\mathrm{CS}+$ than to the CS - , which substantiates that the mixtures are discriminable. As before, the response to all of the intermediate test ratios $(7: 3,5: 5$, $3: 7)$ showed a gradient of decreasing responses from the CS + to the CS - . The opposite slopes of the two gradients $(p<0.01)$ indicates that bees differentiated between CS + and CS - in both cases. 


\section{Excitation and inhibition interact along a perceptual gradient}

The previous set of experiments revealed that discrimination declines as the CS+ and $\mathrm{CS}-$ are made more similar in regard to the molecular composition of the mixtures. This implies that the excitation produced to the CS+ by its association with sucrose reinforcement smoothly declines with progressively larger alterations of the mixture composition. The same applies to a form of "inhibition" generated to the CS - . Therefore, when the CS+ and CSare made more similar they should summate in such a way that the strongest response will be to a mixture that is not the CS+ and which is farther away from the CS-. This phenomenon is called "peak shift" (Spence, 1937; Mackintosh, 1983), and we use it here as a means to imply that transitions across ratios of binary mixtures produce reasonably smooth changes in the perceptual qualities they produce (Daly et al., 2001).

We performed a set of experiments in which bees were differentially conditioned to a 5:5 ratio as the CS+ and to either a $1: 9$ or $9: 1$ ratio as the CS- (supplemental Fig. S1, available at www. jneurosci.org as supplemental material, shows acquisition curves). They were then tested as before with the range of ratios: $1: 9,3: 7,5: 5,7: 3,9: 1$. After conditioning to $5: 5+$ versus $9: 1-$, the strongest response (longest mean duration) was to the 3:7 test ratio ( $p<0.05$ ) (Fig. 2, dashed lines). The direction of the peak shift is consistent with the interaction of excitation and inhibition along a gradient of similarity in the neural codes defined by the test ratios. That is, the response shifted from the $\mathrm{CS}+$ in a direction away from the CS - . However, a peak shift was not evident after conditioning to $5: 5+$ versus 1:9- (Fig. 2, solid lines). The effect in the former condition but not in the latter probably reflects an asymmetry in the coding for these odor ratios (Daly et al., 2001). It is unlikely that the asymmetry was attributable to a preference for $3: 7$, because a preference for that ratio was not evident with conditioning of 9:1 versus 1:9. A more extensive analysis of peak shift in olfactory conditioning of honeybees can be found in the study by Wright et al. (2009b).

In summary, experiments 1 and 2 support the following conclusions, which are important for interpretation of the imaging data below. First, gradations in the ratio of two odors in a mixture produce a smooth generalization from the CS + through the CS - . Second, further support of the graded nature of similarity of mixtures is provided by the peak shift in the response away from 1:9- after 5:5+/1:9- conditioning. The next set of experiments was designed to evaluate how coding for mixtures that grade into one another might be reflected in spatiotemporal patterns set up in the AL.
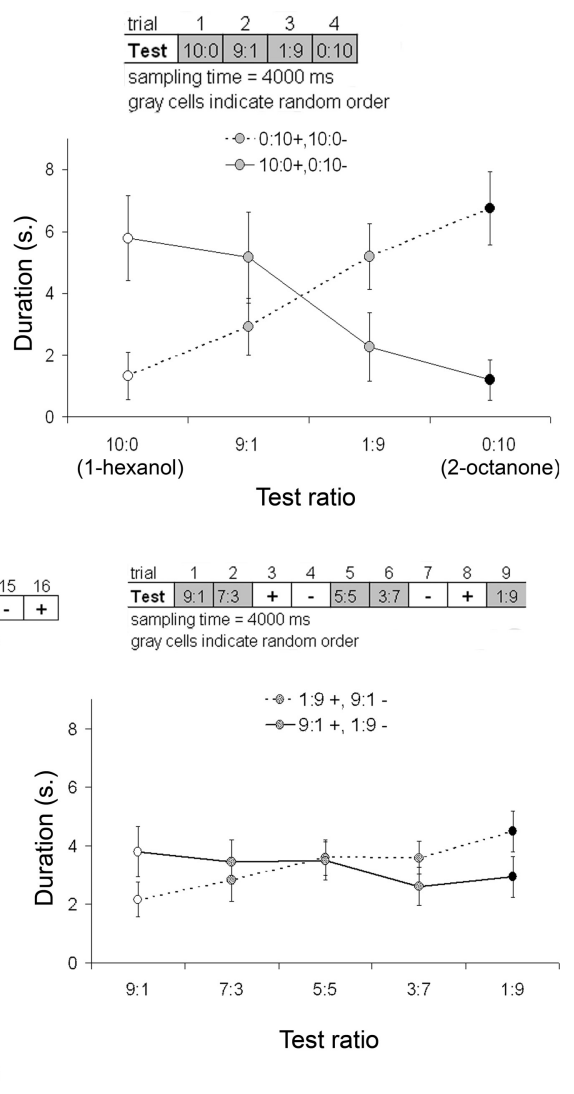

Figure 1. Transitions in ratios of binary mixtures define smooth transitions in odor perception. $\boldsymbol{A}$, Top row of boxes represents CS + and CS - . During testing (gray boxes to the right), different ratios of odors (1-hexanol: 2-octanone) were presented unreforced in a randomized order. The graph shows the duration of the proboscis extension response (see Materials and Methods) sown to the left. Two-way repeated measures ANOVA was performed for the following factors: ratio, NS; conditioning treatment, 作 trials (see Materials and Methods). Left graphs represent percentage proboscis extension during training; retraining trials performed in between recall testing (right) are separated by the gray line. Right graphs represent duration of proboscis extension response during testing (mean $\pm \mathrm{SE}$ ) as a function of different test ratios. Two-way repeated measures ANOVA was performed as above: ratio NS; conditioning treatment, NS; ratio $\times$ treatment $p=0.006, N=28$ bees for $9: 1+, 32$ bees for 1:9+. In all cases, sampling time (odor delivery time) was 4000 ms for both conditioning and testing.

\section{Transient activity patterns show a gradient of activation along different ratios of binary mixtures}

We measured the responses in the AL to binary mixtures along a gradient of ratios from 1-hexanol (10:0) to 2-octanone (0:10). Odor elicited activity in the AL was measured by backfilling PN axons with the calcium indicator fura-2 (see Materials and Methods) (Sachse and Galizia, 2002). The shape and relative position of the glomeruli in the AL is conserved across individuals allowing the identification of individual glomeruli from one animal to the other (Galizia et al., 1999). Since PNs from the l-ACT are stained, the maps of activity obtained with this technique specifically represent the output of an identified set of glomeruli on the dorsal surface of the AL. Calcium activity was recorded in $125 \mathrm{~ms}$ time steps. Although imaging calcium activity is slow relative to electrophysiological measurements (Daly et al., 2004a; Lei et al., 2004; Mazor and Laurent, 2005) and is an indirect indicator of electrical activity, a strong correlation between calcium imaging 


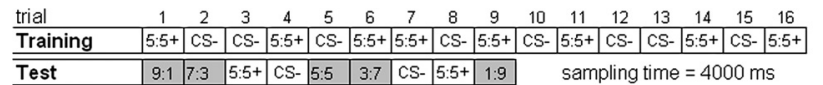

\begin{tabular}{l|l|l|l|l|l|l|l|l|l|l|}
\hline \hline Test & $9: 1$ & $7: 3$ & $5: 5+$ & CS- & $5: 5$ & $3: 7$ & CS- & $5: 5+$ & $1: 9$ \\
\hline
\end{tabular}

gray cells indicate random order

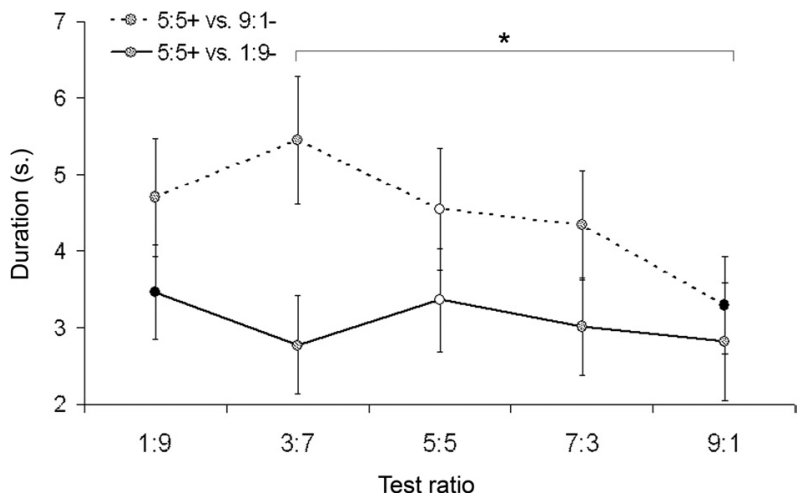

Figure 2. Excitation and inhibition interact to produce to produce a peak shift in the response. Top, Schematic of experimental protocol for bees trained to the $\mathrm{CS}+$ using a 5:5 mixture and $C S-$ either 1:9 or 9:1. Test odors were presented in random order and intermingled with retraining trials. Bottom. Duration of the proboscis extension response for $5: 5+$ versus 9:1 - (dotted line) and 5:5 + versus 1:9 - (full line) as a function of different 1-hexanol: 2-octanone test ratios. Sampling time (odor delivery time) was $4000 \mathrm{~ms}$ for both conditioning and testing. Two-way repeated measures ANOVA was performed for the following factors: test ratio, NS; (GLM a priori contrasts between $3: 7$ and 9:1; $p=0.01$ ); conditioning treatment, NS; interaction ratio $\times$ treatment, $p=0.02$; (GLM a priori contrasts between $3: 7$ and $9: 1 ; p=$ 0.0004 ). (GLM = general linear model). $N=36$ bees for $5: 5+$ versus $1: 9-;$ and $N=39$ bees for $5: 5+$ versus $9: 1-$. Acquisition curves are shown in supplemental Figure $\$ 1$, available at www.jneurosci.org as supplemental material.

and spike rate has been reported using the same calcium sensor (fura-2) and in the same honeybee PNs that we recorded in the present work (Galizia and Kimmerle, 2004).

Figure $3 A$, left panel, shows an example of the raw fluorescence ( $380 \mathrm{~nm}$ excitation; $>510 \mathrm{~nm}$ emission) of an AL $8 \mathrm{~h}$ after staining the ipsilateral 1-ACT axons with fura-2. The homogenous intensity in the raw fluorescence images is an indication of homogenous staining. The bright spots on the medial side of the $\mathrm{AL}$ (left) belong to a cluster of the l-ACT somas (Kirschner et al., 2006) and are also an indication of specific staining. Figure $3 A$, right panel, represents the correlated image of the same AL used to identify glomeruli (see Materials and Methods). Note that dark areas (i.e., low correlation values) do not mean a lack of staining, which is controlled in the raw fluorescence image. Instead, glomeruli in this area were not activated by any of the odors presented to the bee.

Figure $3 B$ shows images representative of activity patterns induced by the seven odors tested. The activity patterns recorded from the different mixtures involved a combination of the PNs (glomeruli) activated by each component, as was previously shown in measurements using a calcium imaging technique in honeybees that most likely reveals activity in olfactory receptor neurons (Deisig et al., 2006). Each odor was measured three times per animal, and the AL response was consistent across these repetitions (see supplemental Fig. S2A,B, available at www. jneurosci.org as supplemental material). A qualitative analysis of the example shown in Figure 3 shows that the PNs differentially excited by one of the pure components were also excited in the mixtures with the highest proportion of that component. Excitation of odor-specific PNs dropped when the relative concentration of that odor decreased. For example, glomerulus 38 (Fig. 3B, upper white circle) is excited by 10:0 (pure 1-hexanol), 9:1 and 7:3. Its activity decreased in 5:5 and vanished in 3:7, 1:9 and 0:10 (pure 2-octanone). Glomerulus 52 (Fig. $3 B$, lower white circle) is strongly activated by $0: 10,1: 9,3: 7$, and $5: 5$, the activation gradually diminishes until 10:0.

A similar analysis in a population of 9 bees showed that, from 17 identified glomeruli, 9 significantly increase activity with increasing concentrations of 2-octanone in the mixture (i.e., glom $17,33,36,42,43,47,48,49,52)$, one significantly increases its activity with increasing concentrations of 1-hexanol (i.e., glom 38 ), and 7 remain invariable (i.e., glom 23, 24, 25, 28, 29, 35, 37) (see examples in Fig. 3C). Therefore, the spatially defined neural representation of binary mixtures among the PNs showed a gradient of activation along the different ratios. At the individual level, some of the glomeruli eventually suggested synergistic or antagonistic interactions along the gradient. For example, visual inspection of Figure $3 B$ shows glomeruli that seem to be more activated by some of the mixture ratios than by the pure components. However, when averaged across animals, none of the glomeruli activated by any of the mixtures showed an obvious and consistent effect of nonadditive interaction.

PN response patterns evolve through time showing a smooth transition along ratios from one pure component to the other We analyzed the temporal evolution of PN activity patterns across the range of odor ratios used in the behavioral experiments. Figure $4 A$ shows the temporal course of an odor signal in selected glomeruli for the ratios 9:1 and 1:9 in an individual animal. Typically, a response is clearly evident by first recorded 125 ms frame after odor onset. As shown in the example in Figure $4 \mathrm{~A}$, different glomeruli showed odor specific amplitudes and different temporal profiles, hence contributing to an odor-specific spatiotemporal response. Most active glomeruli show their peak in activity $\sim 250 \mathrm{~ms}$ after odor onset; however, some glomeruli start to respond later in the odor presentation (e.g., compare glomeruli 36, 48, and 52). Interestingly, some glomeruli may respond early for a given odor and latter for another (see glomerulus 28 for ratios 1:9 and 9:1). The activity decreased abruptly and stabilized back to baseline few hundred milliseconds after odor offset. The bottom panel in Figure $4 A$ shows the temporal evolution of the Euclidean distance (ED) between the odors 1:9 and 9:1 calculated from the examples above, but based on all 17 glomeruli. The distance is maximal at $\sim 500 \mathrm{~ms}$ in this example, which is after the peak activation of many glomeruli at or just after odor. Therefore, the temporal pattern contributes to the separation of the trajectories.

We then analyzed the activity of the 17 identified glomeruli over $125 \mathrm{~ms}$ time steps for each animal. We represented the spatiotemporal patterns as trajectories in a two-dimensional space using Principal Component Analysis. The temporal evolution of the response to each odor is then represented by a trajectory through this space (Fdez Galán et al., 2004; Mazor and Laurent, 2005). Figure $4 B$, left, shows examples of trajectories for the range of odor-ratios presented to two different individuals (bees $\mathrm{C} 1$ and $\mathrm{C} 2$ ). Since this pattern was fairly constant among animals ( 7 of 9 bees showed the same pattern), we averaged the activity of each of the 17 glomeruli along each time point across animals. PCA-based trajectories for the averaged data are shown in Figure $4 B$ (right). After stimulus onset, trajectories depart from rest near the origin and evolve through time (thick line indicates odor exposure). They do not evolve at a constant speed but start to slow down after $500 \mathrm{~ms}$, which is consistent with previous measurements by Fdez Galán et al. (2004). At that point the trajectories start to return although the odor is still being presented. After odor stimulation (dotted line), trajectories return slowly to the 

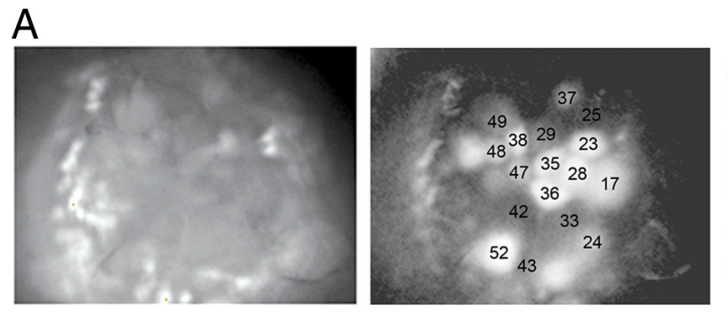

B

1-hexanol 10:0

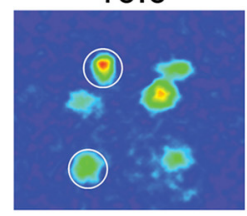

9:1

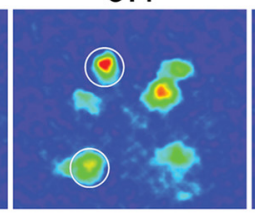

$7: 3$

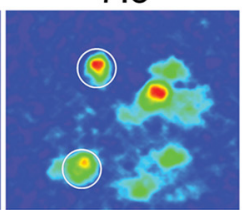

C

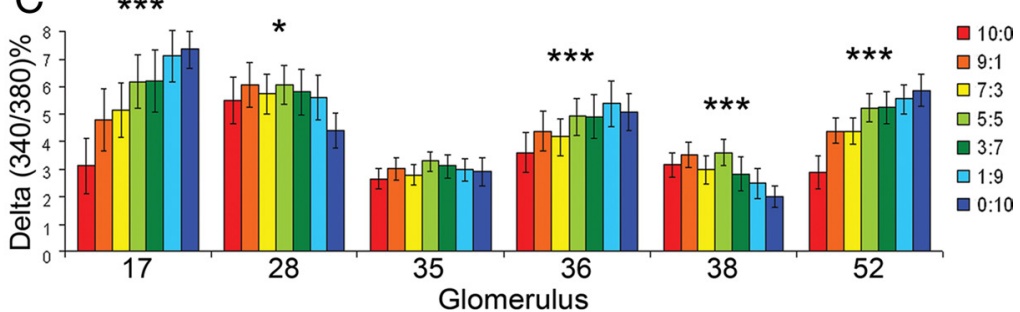

Figure 3. Neural representation of binary mixtures 1-hexanol: 2-octanone in PNs of the AL showed a gradient of activation along different ratios. $\boldsymbol{A}$, Anatomical view (raw fluorescence) of the antennal lobe (left) and correlated image (right) (see Materials and Methods) showing the 17 glomeruli identified in this study. $\boldsymbol{B}$, Color-coded odor response patterns across the dorsal glomeruli in the AL from a representative individual honeybee. PNs were labeled by backfilling with fura 2-dextran. All the ratios from 1-hexanol (10:0) to 2-octanone (0:10) are shown. The maps are represented according to a common intensity scale (right). Each image represents an average of the glomerular activity between 375 and 625 ms after odor onset. C, Glomerular response delta (340/380) percentage of selected individual glomeruli (mean $\pm \mathrm{SE}, N=9$ ) to different ratios of test odors. Mean of glomerular activity between 325 and 625 ms after stimulus onset is shown. In all cases asterisks indicate significant differences by repeated measures ANOVA $\left({ }^{*} p<0.05,{ }^{* * *} p<0.001\right)$.

A
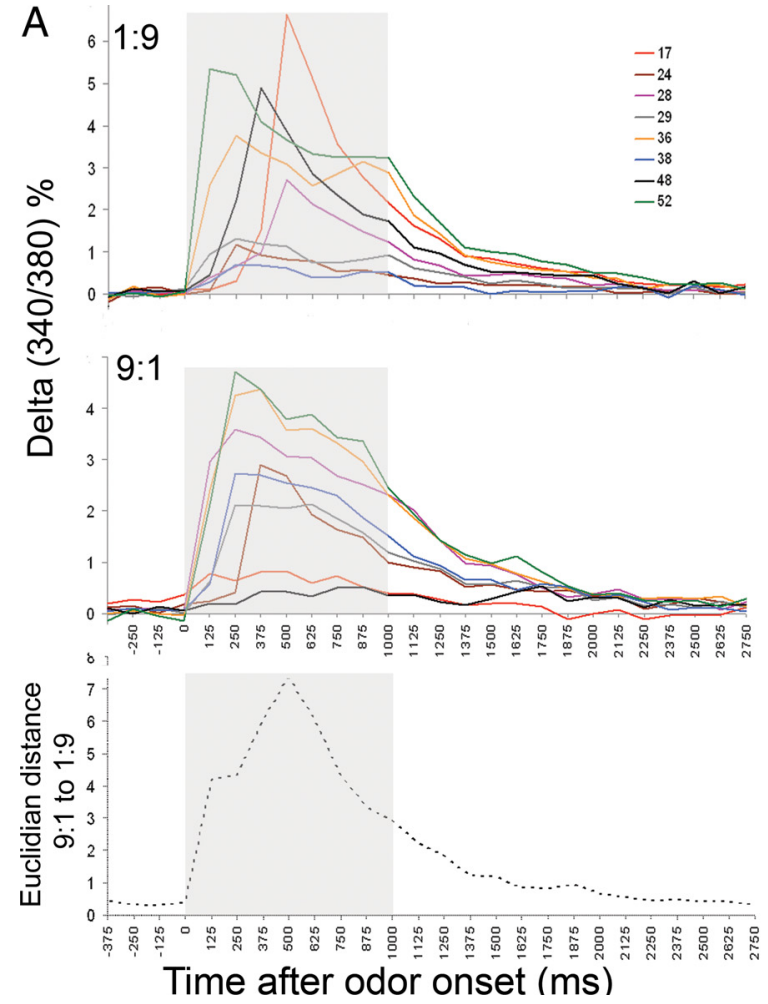

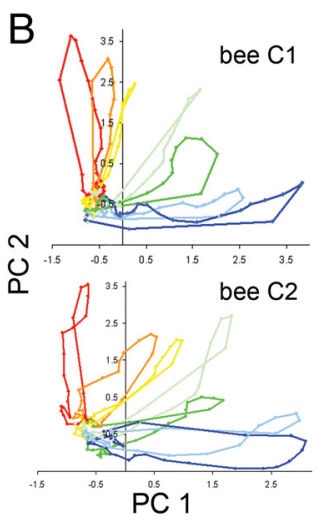

C

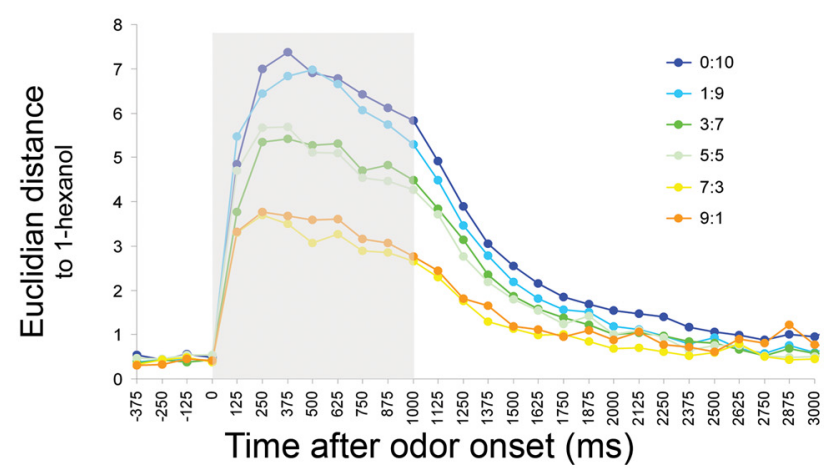

Figure 4. Spatiotemporal response patterns show a smooth transition along ratios from one single component to the other. $\boldsymbol{A}$, Top and middle panel, $0 \mathrm{dor}$ responses (\% delta $340 / 380$ ) in eight representative glomeruli to ratios 9:1 (top) and 1:9 (bottom) over $125 \mathrm{~ms}$ time steps from just before odor delivery through $2750 \mathrm{~ms}$ for a control bee. Different colors correspond to different glomeruli (see legend). Stimulus duration (1000 ms) is marked by the shaded area. Bottom panel, Euclidean distance 9:1 to 1:9 over time for the same control bee. $\boldsymbol{B}$, Left, 0 dor specific trajectories for two control bees; seven of nine bees follow the same general pattern. To generate this figure the original 17-dimensional space has been projected onto the first two principal components for each bee. Under these conditions, $86.2-97.5 \%$ of the variance is explained for each bee. All ratios were presented to each bee and Ca ${ }^{2+}$ transients were recorded at fixed time intervals ( 125 ms). Accordingly, the distance between different colored data points represents the divergence of the odor representations over time. Trajectories depart rapidly from baseline and slow down when they approach odor-specific regions. PC1 and PC2 indicate principal component factors 1 and 2 respectively. Right, PCA of odor-evoked activity patterns for a control "average" bee obtained by averaging 9 control bees by the activity of 17 glomeruli along 27125 ms-time intervals. The response of each glomerulus was used as a dimension for the analysis. $\boldsymbol{C}$, Euclidean distances between ratio $10: 0$ (pure 1-hexanol) and all the other test ratios based on a 17-dimensional space over $125 \mathrm{~ms}$ time steps from just before odor delivery through $3000 \mathrm{~ms}$ after odor onset. Stimulus duration (1000 ms) is marked by the shaded area. 
A

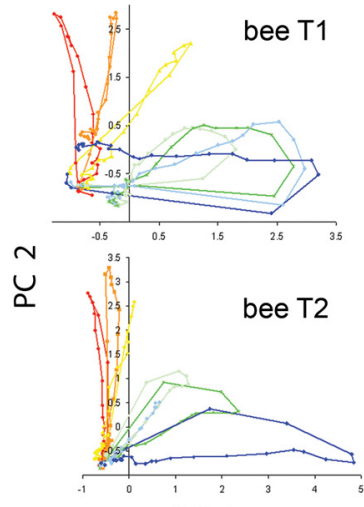

PC 1
$\mathrm{B}$

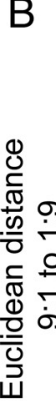

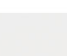

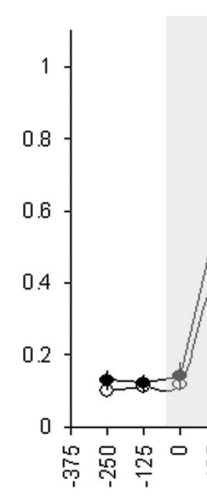

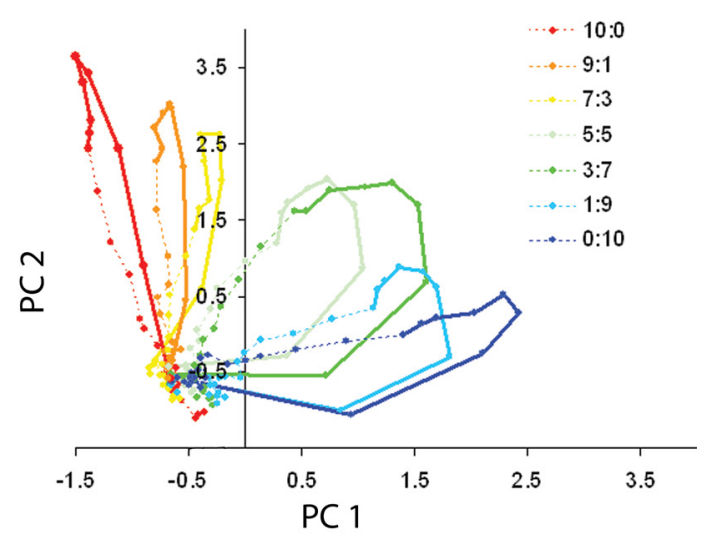

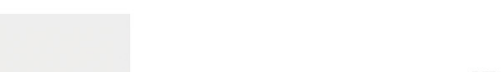

PC

\section{엉} Time after odor onset (ms)

Figure 5. Differential conditioning increases the distance between spatiotemporal patterns. Worker honeybees were differentially trained to 9:1 + and 1:9-. Nine hours after conditioning, brains were treated as above with fura- 2 and $8-12 \mathrm{~h}$ later were imaged as described in Materials and Methods. These bees were evaluated in parallel to the untrained "control" bees reported in Figure 4. PCA analysis was done jointly for untrained and trained animals, allowing a direct comparison of the two groups. $A$, Left, Odor specific trajectories for two trained bees. Seven of eight bees follow the same pattern. The PCA of odor-evoked activity patterns account for $88.2-95.4 \%$ of the variance for each bee. $P(1$ and $P C 2$ indicate principal component factors 1 and 2 respectively. Right, $\mathrm{PCA}$ of odor-evoked activity patterns for a trained average bee $(N=8)$. The relative positions of odor representations between trained and control groups (compare with Fig. 5B, right) suggest the modification of odor representation through conditioning. The first two components account for $97 \%$ of the whole variance of the trajectories. $\boldsymbol{B}$, Relative euclidean distances between ratios 9:1 and 1:9 (i.e., $C S+$ vs $C S-$ ) based on a 17-dimensional space over 125 ms time steps from just before odor delivery through $3000 \mathrm{~ms}$ after odor onset. Stimulus pulse $(1000 \mathrm{~ms})$ is marked by the shaded area. Close and open symbols indicate respectively trained and untrained bees. Asterisks indicate significant differences between trained and control bees (Mann-Whitney $U$ test, $p<0.05$ ).

resting state following a path that is slightly different from the path during stimulation (Mazor and Laurent, 2005).

In addition, the spatiotemporal patterns change in an ordered way as the mixture ratio changes from one extreme to the other. The pure odors (0:10 and 10:0) correspond approximately to each of the PC axes. As the mixture ratio changes from 10:0 to 9:1 to 7:3 through the other pure odor, the angle of the loop through PC space gradually changes (Fig. $4 B$ ). To quantify the relationships between odors along time, we calculated EDs for each time point in the 17-dimensional space represented by the different glomeruli (Fig. 4C). Each ED obtained for each time point in each animal was normalized to its ED obtained between 10:0-0:10 at $500 \mathrm{~ms}$ (see Materials and Methods) (supplemental Fig. S3, available at www.jneurosci.org as supplemental material, also shows a different method for calculating ED).

In summary, after odor onset the distance between trajectories for 10:0 and different ratios begins to depart from baseline by 125 $\mathrm{ms}$ and it is significantly different by $250 \mathrm{~ms}$ (repeated measures ANOVA between 0 and $250 \mathrm{~ms}$, time factor $p<0.001$ ). The maximum odor separation was reached by 375 or $500 \mathrm{~ms}$ (Fig.
$4 A)$. Because the temporal evolution of responses differs in different glomeruli and contributes to the separation, this separation is the temporal decorrelation referred to by Mazor and Laurent (2005). The distance then slowly decreased showing no sharp boundary when the odor was shut off at $1000 \mathrm{~ms}$. The distance stabilizes back to baseline levels by $\sim 800 \mathrm{~ms}$ after odor shut off. The distances between trajectories become progressively greater as the difference in ratios increases from 10: 0-9:1 through 10:0-0:10 (Fig. 4C). The gradient in these temporal patterns of PN responses therefore correlates closely to what we observed in the behavioral assays.

\section{Differential conditioning increased the decorrelation between AL responses to odor mixtures}

The imaging data summarized above comes from untrained honeybee workers. However, all of the previously reported behavioral responses were from trained honeybees. Therefore, we also evaluated AL responses to odors in honeybees that had been differentially conditioned to odor mixtures. We show examples of odor trajectories of two trained individuals (bees T1 and T2) (Fig. 5A, left) as presented before for control animals (Fig. $4 B)$. Again, since this pattern was fairly constant among animals, we averaged the activity of each of the 17 glomeruli along each time point across trained animals. (The PCA in Figs. 5A, right, and $4 B$, right, was done jointly for untrained and trained animals to allow a direct comparison.) Trajectories for the average are shown to the right of Figure $5 \mathrm{~A}$. This visualization of odor trajectories suggests a shift in odor trajectories to the left in trained bees (Figs. 5A, right, $4 B$, right, compare same color lines). Interestingly, the relative distance among odor trajectories for the different ratios showed qualitative changes after differential conditioning. The trajectory for 9:1 (CS+) moved away from 0:10, 1:9 (CS-), 3:7, and 5:5 and became more similar to 10:0, the single component 1-hexanol, which is the major component in the rewarded ratio.

Figure $5 B$ shows the Euclidean distances from -250 to 3000 $\mathrm{ms}$ (125 ms interval) for the comparison between CS+ and CS(9:1 vs 1:9) in trained and untrained animals. The pattern for trained animals (closed symbols) was similar in its time course to that for control, untrained animals (open symbols), but it was different in magnitude. As before, distances departed from baseline by $125 \mathrm{~ms}$. However, the distances were significantly larger in trained than in untrained animals. This difference was significant by $250 \mathrm{~ms}$, and it remained significant at each time point between 375 and $1000 \mathrm{~ms}$ after odor onset (Mann-Whitney $U$ test, $p<$ 0.05 ) (see also supplemental Fig. S3, available at www.jneurosci. org as supplemental material). Therefore, the reference frame for 


\begin{tabular}{|c|c|c|c|c|c|c|c|c|c|c|c|c|c|c|c|c|}
\hline trial & 1 & 2 & 3 & 4 & 5 & 6 & 7 & 8 & 9 & 10 & 11 & 12 & 13 & 14 & 15 & 16 \\
\hline Training & $9: 1+$ & 1:9- & 1:9- & 9:1+ & 1:9- & 9:1+ & $9: 1+$ & $1: 9$ & $9: 1+$ & 1:9- & $9: 1+$ & 1:9- & 1:9- & 9:1+ & 1:9- & $9: 1+$ \\
\hline \multicolumn{17}{|c|}{ sampling time during training (tTR) was fix either $200,500,1000$ or 2000 ms within experiments } \\
\hline Test & 9:1 & $1: 9$ & 9:1+ & 1:9- & $9: 1$ & $1: 9$ & $9: 1+$ & 1:9- & 9:1 & $1: 9$ & sv & $9: 1+$ & $9: 1$ & $1: 9$ & & \\
\hline sampling time & \multicolumn{2}{|c|}{$200 \mathrm{~ms}$} & \multicolumn{2}{|c|}{ tTR } & \multicolumn{2}{|c|}{$500 \mathrm{~ms}$} & \multicolumn{2}{|c|}{ tTR } & \multicolumn{2}{|c|}{$1000 \mathrm{~ms}$} & \multicolumn{2}{|c|}{ tTR } & \multicolumn{2}{|c|}{$2000 \mathrm{~ms}$} & & \\
\hline
\end{tabular}
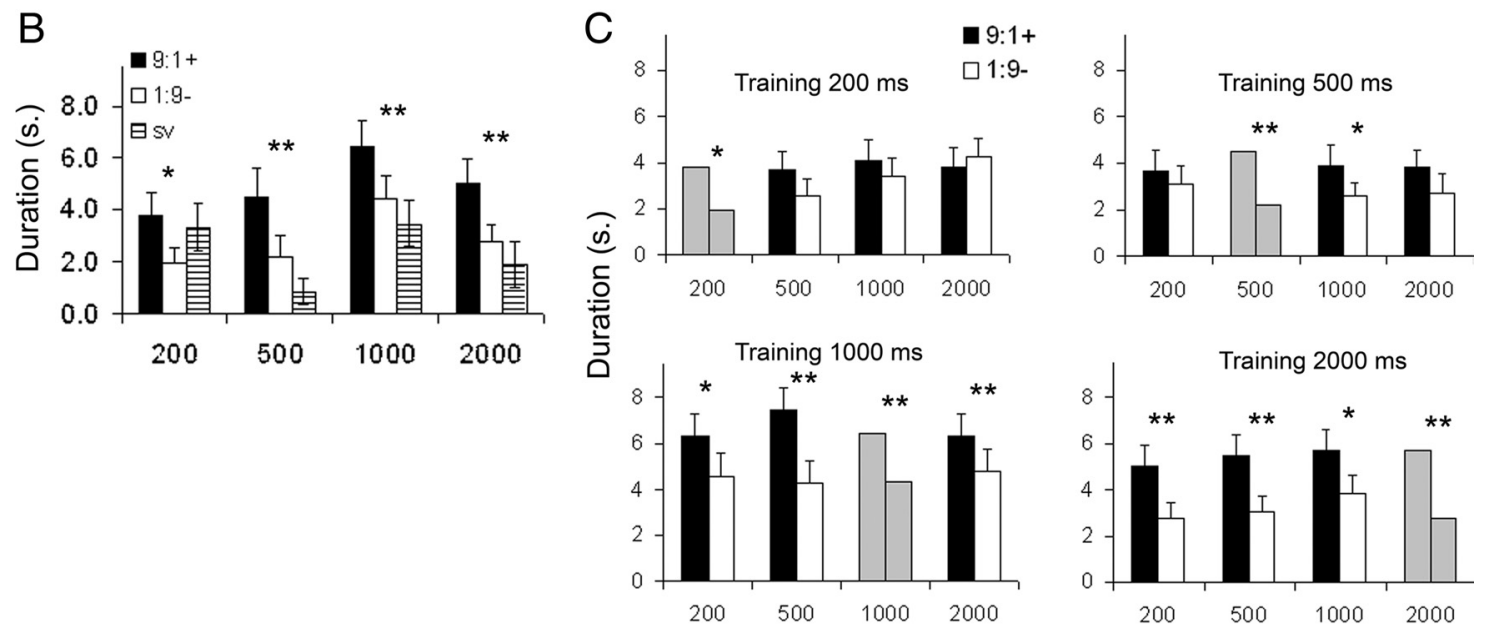

Test time (ms.)

Figure 6. Training times $<500$ ms lead to poor discrimination when test time does not match training time. $A, S$ chematic of experimental protocol for bees trained to CS $+9: 1$ and CS $-1: 9$ (ratio 1-hexanol: 2-octanone). $t_{\mathrm{TR}}$ indicates odor sampling time during training (either 200, 500, 1000, or $2000 \mathrm{~ms}$ ). During testing trials, the odors were presented in a random sequence. SV indicates stimulation by solvent. Thus, each set of honeybees was trained using one sampling time and then tested with each of the four times. Acquisition curves for each set are shown in supplemental Figure S2, available at www.jneurosci.org as supplemental material. Sample sizes were: $N=37$ for $200 \mathrm{~ms}, N=30$ for $500 \mathrm{~ms}, N=39$ for $1000 \mathrm{~ms}$, and $N=40$ for $2000 \mathrm{~ms}$. $\boldsymbol{B}$, Duration of the proboscis extension response when odor sampling time during training matched test time, i.e., honeybees were trained and tested with the same sampling time. Hatched bars indicate response levels during the test with $10 \mu$ l of solvent (mineral oil). C, Duration of the conditioned response for all combinations of training and testing times (i.e., bees were trained with a specific stimulus length and tested with the same or with a different one). Gray bars denote data already shown in Figure $3 B$ (presented here for comparison). In all cases, asterisks indicate significant differences by Wilcoxon matched pairs test between $9: 1$ and $1: 9\left({ }^{*} p<0.05,{ }^{* *} p<0.005\right)$.

the PN coding space was shifted in trained bees in a way that improves the separation between the CS + and CS-.

Changes in activity across glomeruli were complex. As a result of differential conditioning, some glomeruli, for example 25, became slightly more excited, and other glomeruli (e.g., 17, 33, 36, 48 , and 52) showed a slight reduction in activity (supplemental Fig. S4, available at www.jneurosci.org as supplemental material). However, all of these changes were subtle and not significant in any case, suggesting that differences in EDs as shown in Figure $5 B$ are not dominated by the differential activation of one or two specific glomeruli.

Training times $<500 \mathrm{~ms}$ increase generalization when the test time does not match the training time

We have shown that the representation of odors in the AL is dynamic and depends on sampling time (Fdez Galán et al., 2004; Mazor and Laurent, 2005). We therefore performed a series of experiments to evaluate how temporal information may be involved in odor recognition. We also included a dimension not yet tested in olfactory conditioning experiments, which consists of matching or mismatching sampling times during training and testing. If the pattern at a particular time is stored in memory during training, then animals may or may not be capable of recognizing stimuli given different sampling times during recall tests.

In this experiment, we used the same odor ratios 9:1+ versus 1:9- and four different stimulus delivery times (200, 500, 1000, and $2000 \mathrm{~ms}$ ) (Fig. 6A). These delivery times are realistic times that honeybees might encounter when flying upwind in an odor plume in the field (Vickers, 2006). Sensory afference from the antennae, as measured by electroantennogram responses, reaches a maximum by $200 \mathrm{~ms}$ (supplemental Fig. S5 $A, B$, available at www.jneurosci.org as supplemental material), which indicates that the different sampling times that we used are probably not perceived as different odor concentrations (Stopfer et al., 2003). Furthermore, short stimulation times produced activity patterns across glomeruli in the AL that are nested within the patterns produced by longer stimulation (supplemental Fig. S5C, available at www.jneurosci.org as supplemental material). Therefore, different sampling times are likely represented as truncated trajectories in the 17-dimensional space (Mazor and Laurent, 2005). In most cases, honeybees began to respond more to the 9:1+ ratio than to the $1: 9-$ by the third or fourth trial, and this pattern carried through the testing phase (supplemental Fig. S6, available at www.jneurosci.org as supplemental material).

Figure $6 B$ shows the duration of proboscis extension when training time matched testing time. The mean duration was higher for the CS + than for the CS - in all cases $(p<0.05$; Wilcoxon matched pairs test), indicating that honeybees were able to discriminate test ratios even with only $200 \mathrm{~ms}$ exposure. However, two patterns indicate that exposure time is important for discriminability. First, the duration of response (mean of the $\mathrm{CS}+$ and CS - response levels) increased from $3.3 \mathrm{~s}$ or $3.4 \mathrm{~s}$ in the 200 and $500 \mathrm{~ms}$ stimulation groups, to $5.5 \mathrm{~s}$ in the $1000 \mathrm{~ms}$ group (one-way ANOVA, $p=0.02$ ). This increase in response likely indicates that long sampling times increase the detectability of 
odor over background, as was already reported by Wright et al. (2009a). Another important indication of a changing pattern of response comes from comparison to test responses to the solvent blank. At $500 \mathrm{~ms}$ or longer training/test exposures, the responses to the CS+ were on average longer than the mean responses to the solvent blanks (Fig. $6 B$, hatched bars) $(p<0.001)$. However, at $200 \mathrm{~ms}$ the response to the blank was equal to the CS+ and qualitatively longer than the responses to the CS-. This change in pattern suggests that honeybees may adopt different response strategies depending on sampling time. For example, the CSmay become discriminable from the blank much more quickly than the CS+. Regardless of the underlying cause, this result suggests that sampling time is a determinant of the response topology and decision strategy.

Further evidence that sampling time is important came from testing with times that were different-either shorter or longerthan training times (Fig. 6C). When honeybees were trained with $200 \mathrm{~ms}$ exposure, bees did not differ in response to the CS + and CS - when odors were delivered at any of the times longer than $200 \mathrm{~ms}$. After training to $500 \mathrm{~ms}$, the highest generalization occurred at the shortest (200 ms) and at the longest (2000 ms) test exposures. The only significant indication of discrimination occurred when there was a match $(500-500 \mathrm{~ms})$ or a slight mismatch (500-1000 ms) between training and test exposures. Finally, discrimination was evident at all testing times with training exposures of 1000 or $2000 \mathrm{~ms}$.

Animals that responded to odorants did so with approximately the same latency. The median latencies for the CS + and CS - were $600 \mathrm{~ms}$. Means were, respectively, $740 \mathrm{~ms}( \pm 50 \mathrm{~ms}$, $\mathrm{SE})$ and $810 \mathrm{~ms}( \pm 70 \mathrm{~ms}, \mathrm{SE})$. These values did not differ significantly across sampling time $(p>0.05$ for all comparisons among 200, 500, 1000, and $2000 \mathrm{~ms}$ ).

\section{Discussion}

We have shown that the transient dynamics in the PN output of the AL represent gradations of odor mixtures that correlate to behavioral generalization among those mixtures. Discrimination conditioning of binary mixtures produced smooth generalization during testing with intermediate ratios. The response to a test odor was directly proportional to its similarity to the CS+ or CS - . Furthermore, conditioning odor ratios that were similar produced a peak shift in the response in a direction away from the CS-. All of the behavior data suggest that smooth changes in mixture ratio produce smooth changes in the perceptual properties and, hence, in the neural representations of those mixtures. Therefore, responses in the AL should also grade smoothly with systematic variation in the ratio of components. Such smooth gradations in spatial response patterns have been shown in several studies of molecular features of monomolecular odorants (Sachse et al., 1999; Daly et al., 2004a; Lei et al., 2004; Hallem and Carlson, 2006; Johnson and Leon, 2007). Smooth gradations to changes in odor mixture ratio have been shown in the mammal OB (Khan et al., 2008) and insects (Carlsson et al., 2007). However, little work has been done to investigate spatiotemporal coding using systematic changes in ratios of mixtures.

We show that the orientation of the transient's trajectory changes systematically with the composition of the mixture. As the ratios became more distinct the loops became farther separated, which correlates to generalization among odors in behavioral studies. The mechanism through which spatiotemporal patterns generate this gradation remains to be investigated. Several studies of spatial coding in sensory afferents have described mixture coding in honeybees (Deisig et al., 2006), fruit flies (Hallem and Carlson, 2006; Silbering and Galizia, 2007) and moths (Carlsson et al., 2007). In general, mixture-induced activity patterns include patterns of each component. Complex mixtures largely activate patterns that contain component patterns, but with some suppression possibly because of global inhibitory interactions among glomeruli (Deisig et al., 2006).

Our studies investigated mixture coding in the output of AL. We found no obvious evidence of synergism or suppression. The lack of significant interaction between glomeruli activated by each of the odors could explain the smooth, almost additive transition of spatiotemporal pattern that we observed. This finding is consistent with a recent report on mixture coding in the mammalian OB (Khan et al., 2008). In contrast, Silbering and Galizia (2007) reported mixture suppression in PNs in Drosophila. Activated glomeruli suppressed other glomeruli through networklevel inhibitory connections (Leitch and Laurent, 1996; Wilson and Laurent, 2005; Sachse et al., 2006; Mwilaria et al., 2008). Our study was not designed to specifically reveal interaction. Thus, a more specific, detailed analysis of mixture interaction will be necessary to establish how changes in mixture composition account for smooth changes in spatiotemporal activity.

Several studies have shown associative plasticity in the $\mathrm{OB}$ and $\mathrm{AL}$ (for review, see Davis, 2004). The most frequent change is in recruitment of units into a preexisting pattern (Kendrick et al., 1997; Faber et al., 1999; Ravel et al., 2003; Martin et al., 2004; Yu et al., 2004). Other studies have reported more complex changes involving many or all of the possible types of switches in activity between no response, excitation, and inhibition (Kay and Laurent, 1999; Daly et al., 2004b). The changes reported have been transient, lasting a few minutes in support of a form of short-term memory (Yu et al., 2004); they have lasted up to $3 \mathrm{~h}$ in support of an intermediate form of memory in the AL (Daly et al., 2004b); or they appear to be stable for much longer periods of time (Brennan and Keverne, 1997; Kendrick et al., 1997; Ravel et al., 2003; Sandoz et al., 2003; Martin et al., 2004). It is unclear whether these differences reflect choice of animal model, conditioning protocol, or recording technique.

We found that activity patterns of PNs are more distinct in trained than in control animals, which suggests that the AL is becoming tuned to best discriminate odors that are currently important (Smith et al., 2006). The change lasted at least $24 \mathrm{~h}$, or it developed over that time, because animals were conditioned on the day before imaging studies. Thus a component of associative olfactory memory is consolidated in the AL, which is consistent with biochemical analyses of honeybee and fruit fly AL (Müller, 2000; Ashraf et al., 2006).

In a recent work in honeybees, Peele et al. (2006) used the same recording technique that we have used-backfilling of PNs with fura-dextran. However, they could not identify traces of associative plasticity in the projection neurons of the same tract that we studied here (l-ACT). There are several significant differences between our study and theirs that may explain the different outcomes and provide important insight into how plasticity operates in the antennal lobes. First, Peele et al. (2006) evaluated glomerular activity patterns up to $23 \mathrm{~min}$ after conditioning. We evaluated the activity patterns $24 \mathrm{~h}$ after conditioning. Memory traces at these two time points require different biochemical mechanisms (Müller, 2000) and may involve different cellular substrates (Erber et al., 1980; Hammer and Menzel, 1995, 1998). Our results would be consistent with those of Peele et al. (2006) if the presence of a memory trace in the l-ACT PNs emerges as memory consolidates and is either not present or not easily measurable using calcium imaging during short-term memory. 
Our study also differed from the one by Peele et al. (2006) in the way in which reinforcement was delivered. They conditioned honeybees after PNs had been stained and the animals had been prepared for imaging. In this situation, the US is delivered to the sucrose receptors on the antennae and proboscis, but the animal may not be able to fully consume the sucrose. Stimulation of taste receptors is enough to induce short-term memory, but consumption of sucrose, as honeybees were allowed to do in our study, is an important condition for induction of long-term memory formation (Wright et al., 2007).

Instead of using pure odors that are easily to discriminate, we required animals to differentiate between binary mixtures that differ in the ratio of the two components. Use of mixtures under these conditions might prolong the time needed to effectively differentiate different odors. For example, Krofczik et al. (2008) used pure odors and found that maximal separation occurred circa 100-200 ms after onset of odor. Furthermore, the two components in our experiments were chosen because they activate a partially overlapping set of glomeruli (Sachse et al., 1999). These odor mixtures induce in the AL complex patterns that are similar in untrained animals. Learning different predictive values of two mixtures constitutes a difficult conditioning task, because successful discrimination requires more trials than when conditioning to relatively distinct pure odors (Smith and Menzel, 1989). We propose that in this situation the AL extracts different features of a mixture that more reliably predict reward, making both representations more different than in untrained animals (Smith et al., 2006). This phenomenon may be less evident between pure odors that have little or no overlap in their AL activity patterns.

We also established that stimulus duration is important for discriminability, as measured in generalization gradients. However, it is not a simple rule, such as that more time means better discrimination. If we had only tested stimulus durations that matched training, we might have concluded that stimulus duration is not relevant to odor discrimination, since animals showed discrimination even with very short $(200 \mathrm{~ms})$ stimulus pulses. We found a threshold for stimulus durations below which matching between training and testing is necessary for robust odor discrimination. For short training stimuli of $200 \mathrm{~ms}$, odors were discriminable only when there was a match between training conditions, under which the memory is formed, and test conditions under which the stimulus is compared with the memory template. For longer training stimuli of 1000 or 2000 $\mathrm{ms}$, discriminability became independent of stimulus duration. When training stimuli lasted $500 \mathrm{~ms}$, we observed a transition in the matching requirement, because discrimination was possible with testing stimuli of 500 and $1000 \mathrm{~ms}$. This shift in memory content-from time dependent to time invariant-occurs between 500 and $1000 \mathrm{~ms}$ after odor onset, which is approximately the time it takes for the spatiotemporal patterns to become maximally separated in the imaging analysis.

In natural environments the duration of odor pulses may be short and stochastic (Murlis and Jones, 1981), and it is likely that this would produce variable length pulses that do not match memory. However, animals may behaviorally compensate for this limitation. When allowed a choice, freely flying honeybees fly over an odor source for of $690 \mathrm{~ms}$ regardless of the difficulty of the task (Ditzen et al., 2003). We also found that the median latency to response to the CS+ and CS - was just over $700 \mathrm{~ms}$. According to our imaging measurements, this is beyond the time necessary for the odor representation to reach maximal distinctiveness, and it is likely beyond the time at which odor discrimination becomes time invariant. This suggests that bees make decisions only after having obtained enough information to generate a more reliable encoding of the stimulus.

Finally, the plasticity we have described is consistent with predictions of recent computational models of the AL (Linster and Smith, 1997; Borisyuk and Smith 2004). In those models, reinforcement may modify local inhibitory (GABAergic) synapses. Further work is now needed to test for this specific connectivity in the $\mathrm{AL}$, particularly in regard to disruption of octopamine receptor pathways in the honeybee AL (Farooqui et al., 2003, 2004).

\section{References}

Abel R, Rybak J, Menzel R (2001) Structure and response patterns of olfactory interneurons in the honeybee, Apis mellifera. J Comp Neurol 437:363383.

Abraham NM, Spors H, Carleton A, Margrie TW, Kuner T, Schaefer AT (2004) Maintaining accuracy at the expense of speed: stimulus similarity defines odor discrimination time in mice. Neuron 44:865-876.

Ashraf SI, McLoon AL, Sclarsic SM, Kunes S (2006) Synaptic protein synthesis associated with memory is regulated by the RISC pathway in Drosophila. Cell 124:191-205.

Beshel J, Kopell N, Kay LM (2007) Olfactory bulb gamma oscillations are enhanced with task demands. J Neurosci 27:8358-8365.

Bitterman ME, Menzel R, Fietz A, Schäfer S (1983) Classical conditioning of proboscis extension in honeybees (Apis mellifera). J Comp Psychol 97:107-119.

Borisyuk A, Smith BH (2004) Odor interactions and learning in a model of the insect antennal lobe. Neurocomputing 58-60:1041-1047.

Brennan PA, Keverne EB (1997) Neural mechanisms of mammalian olfactory learning. Prog Neurobiol 51:457-481.

Carlsson MA, Chong KY, Daniels W, Hansson BS, Pearce TC (2007) Component information is preserved in glomerular responses to binary odor mixtures in the moth Spodoptera littoralis. Chem Senses 32:433-443.

Daly KC, Chandra S, Durtschi ML, Smith BH (2001) The generalization of an olfactory-based conditioned response reveals unique but overlapping odour representations in the moth Manduca sexta. J Exp Biol 204: 3085-3095.

Daly KC, Wright GA, Smith BH (2004a) Molecular features of odorants systematically influence slow temporal responses across clusters of coordinated antennal lobe units in the moth Manduca sexta. J Neurophysiol 92:236-254.

Daly KC, Christensen TA, Lei H, Smith BH, Hildebrand JG (2004b) Learning modulates the ensemble representations for odors in primary olfactory networks. Proc Natl Acad Sci U S A 101:10476-10481.

Davis RL (2004) Olfactory learning. Neuron 44:31-48.

Deisig N, Giurfa M, Lachnit H, Sandoz JC (2006) Neural representation of olfactory mixtures in the honeybee antennal lobe. Eur J Neurosci 24:1161-1174.

Ditzen M, Evers JF, Galizia CG (2003) Odor similarity does not influence the time needed for odor processing. Chem Senses 28:781-789.

Duchamp-Viret P, Duchamp A, Chaput MA (2003) Single olfactory sensory neurons simultaneously integrate the components of an odour mixture. Eur J Neurosci 18:2690-2696.

Erber J, Masuhr TH, Menzel R (1980) Localization of short-term memory in the brain of the bee, Apis mellifera. Physiol Entomol 5:343-358.

Faber T, Joerges J, Menzel R (1999) Associative learning modifies neural representations of odors in the insect brain. Nat Neurosci 2:74-78.

Farooqui T, Robinson K, Vaessin H, Smith BH (2003) Modulation of early olfactory processing by an identified octopaminergic reinforcement pathway in the honeybee. J Neurosci 23:5370-5380.

Farooqui T, Vaessin H, Smith BH (2004) Octopamine receptors in the honeybee (Apis mellifera) brain and their disruption by RNA-mediated interference. J Insect Physiol 50:701-713.

Fdez Galán R, Sachse S, Galizia CG, Herz AV (2004) Odor-driven attractor dynamics in the antennal lobe allow for simple and rapid olfactory pattern classification. Neural Comput 16:999-1012.

Flanagan D, Mercer AR (1989) An atlas and 3-D reconstruction of the antennal lobes in the worker honey bee, Apis mellifera L. (Hymenoptera: apidae). Int J Insect Morphol Embryol 18:145-159.

Fletcher ML, Wilson DA (2003) Olfactory bulb mitral-tufted cell plasticity: 
odorant-specific tuning reflects previous odorant exposure. J Neurosci 23:6946-6955.

Friedrich RW, Laurent G (2004) Dynamics of olfactory bulb input and output activity during odor stimulation in zebrafish. J Neurophysiol 91:2658-2669.

Friedrich RW, Habermann CJ, Laurent G (2004) Multiplexing using synchrony in the zebrafish olfactory bulb. Nat Neurosci 7:862-871.

Galizia CG, Kimmerle B (2004) Physiological and morphological characterization of honeybee olfactory neurons combining electrophysiology, calcium imaging and confocal microscopy. J Comp Physiol A Neuroethol Sens Neural Behav Physiol 190:21-38.

Galizia CG, McIlwrath SL, Menzel R (1999) A digital three-dimensional atlas of the honeybee antennal lobe based on optical sections acquired by confocal microscopy. Cell Tissue Res 295:383-394.

Giraudet P, Berthommier F, Chaput M (2002) Mitral cell temporal response patterns evoked by odor mixtures in the rat olfactory bulb. J Neurophysiol 88:829-838.

Giurfa M (2007) Behavioral and neural analysis of associative learning in the honeybee: a taste from the magic well. J Comp Physiol A Neuroethol Sens Neural Behav Physiol 193:801-824.

Hallem EA, Carlson JR (2006) Coding of odors by a receptor repertoire. Cell 125:143-160

Hammer M, Menzel R (1995) Learning and memory in the honeybee. J Neurosci 15:1617-1630.

Hammer M, Menzel R (1998) Multiple sites of associative odor learning as revealed by local brain microinjections of octopamine in honeybees. Learn Mem 5:146-156.

Heinbockel T, Christensen TA, Hildebrand JG (2004) Representation of binary pheromone blends by glomerulus-specific olfactory projection neurons. J Comp Physiol A Neuroethol Sens Neural Behav Physiol 190:1023-1037.

Johnson BA, Leon M (2000) Modular representations of odorants in the glomerular layer of the rat olfactory bulb and the effects of stimulus concentration. J Comp Neurol 422:496-509.

Johnson BA, Leon M (2007) Chemotopic odorant coding in a mammalian olfactory system. J Comp Neurol 503:1-34.

Kashiwadani H, Sasaki YF, Uchida N, Mori K (1999) Synchronized oscillatory discharges of mitral/tufted cells with different molecular receptive ranges in the rabbit olfactory bulb. J Neurophysiol 82:1786-1792.

Kay LM, Laurent G (1999) Odor- and context-dependent modulation of mitral cell activity in behaving rats. Nat Neurosci 2:1003-1009.

Kendrick KM, Guevara-Guzman R, Zorrilla J, Hinton MR, Broad KD, Mimmack M, Ohkura S (1997) Formation of olfactory memories mediated by nitric oxide. Nature 388:670-674.

Khan AG, Thattai M, Bhalla US (2008) Odor representations in the rat olfactory bulb change smoothly with morphing stimuli. Neuron 57:571-585.

Kirschner S, Kleineidam CJ, Zube C, Rybak J, Grünewald B, Rössler W (2006) Dual olfactory pathway in the honeybee, Apis mellifera. J Comp Neurol 499:933-952.

Krofczik S, Menzel R, Nawrot MP (2008) Rapid odor processing in the honeybee antennal lobe network. Front Comput Neurosci 2:1-13.

Laurent G (2002) Olfactory network dynamics and the coding of multidimensional signals. Nat Rev Neurosci 3:884-895.

Laurent G, Stopfer M, Friedrich RW, Rabinovich MI, Volkovskii A, Abarbanel HD (2001) Odor encoding as an active, dynamical process: experiments, computation, and theory. Annu Rev Neurosci 24:263-297.

Lei H, Christensen TA, Hildebrand JG (2004) Spatial and temporal organization of ensemble representations for different odor classes in the moth antennal lobe. J Neurosci 24:11108-11119.

Leitch B, Laurent G (1996) GABAergic synapses in the antennal lobe and mushroom body of the locust olfactory system. J Comp Neurol 372:487-514.

Linster C, Smith BH (1997) A computational model of the response of honey bee antennal lobe circuitry to odor mixtures: overshadowing, blocking and unblocking can arise from lateral inhibition. Behav Brain Res $87: 1-14$.

Mackintosh NJ (1983) Conditioning and associative learning. New York: Oxford UP.

Martin C, Gervais R, Hugues E, Messaoudi B, Ravel N (2004) Learning modulation of odor-induced oscillatory responses in the rat olfactory bulb: a correlate of odor recognition? J Neurosci 24:389-397.

Mauelshagen J (1993) Neural correlates of olfactory learning paradigms in an identified neuron in the honeybee brain. J Neurophysiol 69:609-625.

Mazor O, Laurent G (2005) Transient dynamics versus fixed points in odor representations by locust antennal lobe projection neurons. Neuron 48:661-673.

Meister M, Bonhoeffer T (2001) Tuning and topography in an odor map on the rat olfactory bulb. J Neurosci 21:1351-1360.

Menzel R (1990) Learning, memory and "cognition" in honeybees. In Neurobiology of comparative cognition (Kesner RP, Olton DS eds), pp 237292. Hillsdale, NJ: Erlbaum.

Müller U (2000) Prolonged activation of cAMP-dependent protein kinase during conditioning induces long-term memory in honeybees. Neuron $27: 159-168$.

Murlis J, Jones CD (1981) Fine-scale structure of odor plumes in relation to insect orientation to distant pheromones and other attractant sources. Physiol Entomol 6:71-86.

Mwilaria EK, Ghatak C, Daly KC (2008) Disruption of GABA A in the insect antennal lobe generally increases odor detection and discrimination thresholds. Chem Senses 33:267-281.

Peele P, Ditzen M, Menzel R, Galizia CG (2006) Appetitive odor learning does not change olfactory coding in a subpopulation of honeybee antennal lobe neurons. J Comp Physiol A Neuroethol Sens Neural Behav Physiol 192:1083-1103.

Rabinovich M, Huerta R, Laurent G (2008) Neuroscience: Transient dynamics for neural processing. Science 321:48-50.

Ravel N, Chabaud P, Martin C, Gaveau V, Hugues E, Tallon-Baudry C, Bertrand O, Gervais R (2003) Olfactory learning modifies the expression of odourinduced oscillatory responses in the gamma $(60-90 \mathrm{~Hz})$ and beta $(15-40 \mathrm{~Hz})$ bands in the rat olfactory bulb. Eur J Neurosci 17:350-358.

Rinberg D, Koulakov A, Gelperin A (2006) Speed-accuracy tradeoff in olfaction. Neuron 51:351-358.

Rybak J, Menzel R (1993) Anatomy of the mushroom bodies in the honey bee brain: The neuronal connections of the alpha-lobe. J Comp Neurol 334:444-465.

Sachse S, Galizia CG (2002) Role of inhibition for temporal and spatial odor representation in olfactory output neurons: a calcium imaging study. J Neurophysiol 87:1106-1117.

Sachse S, Galizia CG (2003) The coding of odour-intensity in the honeybee antennal lobe: local computation optimizes odour representation. Eur J Neurosci 18:2119-2132.

Sachse S, Rappert A, Galizia CG (1999) The spatial representation of chemical structures in the antennal lobe of honeybees: steps towards the olfactory code. Eur J Neurosci 11:3970-3982.

Sachse S, Peele P, Silbering AF, Gühmann M, Galizia CG (2006) Role of histamine as a putative inhibitory transmitter in the honeybee antennal lobe. Front Zool 3:22.

Sandoz JC, Galizia CG, Menzel R (2003) Side-specific olfactory conditioning leads to more specific odor representation between sides but not within sides in the honeybee antennal lobes. Neuroscience 120:1137-1148.

Silbering AF, Galizia CG (2007) Processing of odor mixtures in the Drosophila antennal lobe reveals both global inhibition and glomerulus-specific interactions. J Neurosci 27:11966-11977.

Silbering AF, Okada R, Ito K, Galizia CG (2008) Olfactory information processing in the Drosophila antennal lobe: anything goes? J Neurosci 28:13075-13087.

Smith BH (1997) An analysis of blocking in odorant mixtures: an increase but not a decrease in intensity of reinforcement produces unblocking. Behav Neurosci 111:57-69.

Smith BH (1998) Analysis of interaction in binary odorant mixtures. Physiol Behav 65:397-407.

Smith BH, Menzel R (1989) The use of electromygram recordings to quantify odourant discrimination in the honey bee, Apis mellifera. J Insect Physiol 35:369-375.

Smith BH, Wright GA, Daly KC (2006) Learning based recognition and discrimination of floral odors. In: Biology of floral scent (Dudareva N Pichersky E, eds), pp 263-295. Boca Raton, FL: CRC.

Spence KW (1937) The differential response in animals to stimuli varying within a single dimension. Psychol Rev 44:430-444.

Stopfer M, Laurent G (1999) Short-term memory in olfactory network dynamics. Nature 402:664-668.

Stopfer M, Bhagavan S, Smith BH, Laurent G (1997) Impaired odour discrimination on desynchronization of odour-encoding neural assemblies. Nature 390:70-74

Stopfer M, Jayaraman V, Laurent G (2003) Intensity versus identity coding in an olfactory system. Neuron 39:991-1004. 
Tabor R, Yaksi E, Weislogel JM, Friedrich RW (2004) Processing of odor mixtures in the zebrafish olfactory bulb. J Neurosci 24:6611-6620.

Thum AS, Jenett A, Ito K, Heisenberg M, Tanimoto H (2007) Multiple memory traces for olfactory reward learning in Drosophila. J Neurosci 27:11132-11138.

Uchida N, Mainen ZF (2003) Speed and accuracy of olfactory discrimination in the rat. Nat Neurosci 6:1224-1229.

Vanderwolf CH, Zibrowski EM (2001) Pyriform cortex beta-waves: odorspecific sensitization following repeated olfactory stimulation. Brain Res 892:301-308.

Vickers NJ (2006) Winging it: moth flight behavior and responses of olfactory neurons are shaped by pheromone plume dynamics. Chem Senses 31:155-166.

Wang JW, Wong AM, Flores J, Vosshall LB, Axel R (2003) Two-photon calcium imaging reveals an odor-evoked map of activity in the fly brain. Cell 112:271-282.

Wilson RI, Laurent G (2005) Role of GABAergic inhibition in shaping odorevoked spatiotemporal patterns in the Drosophila antennal lobe. J Neurosci 25:9069-9079.
Wright GA, Smith BH (2004) Different thresholds for detection and discrimination of odors in the honey bee (Apis mellifera). Chem Senses 29:127-135.

Wright GA, Mustard JA, Kottcamp SM, Smith BH (2007) Olfactory memory formation and the influence of reward pathway during appetitive learning by honey bees. J Exp Biol 210:4024-4033.

Wright GA, Kottcamp SM, Thomson MG (2008) Generalization mediates sensitivity to complex odor features in the honeybee. PLoS ONE 3:e1704.

Wright GA, Carlton M, Smith BH (2009a) A honeybee's ability to learn, recognize, and discriminate odours depends upon odour sampling time and concentration. Behav Neurosci 123:36-43.

Wright GA, Choudhary AF, Bentley MA (2009b) Reward quality influences the development of learned olfactory biases in honeybees. Proc Biol Sci 276:2597-2604.

Yu D, Ponomarev A, Davis RL (2004) Altered representation of the spatial code for odors after olfactory classical conditioning; memory trace formation by synaptic recruitment. Neuron 42:437-449. 